\title{
Development of multiform ventricular tachycardia during atrioventricular nodal reentrant tachycardia
}

\author{
DAVID H BENNETT, * CORMAC COYLE $\dagger$ \\ From the ${ }^{\star}$ Regional Cardiac Centre, Wythenshawe Hospital, Manchester and $†$ Leighton Hospital, Crewe, \\ Cheshire
}

SUMMARY A woman of 18 presented with a supraventricular tachycardia, subsequently shown to be caused by atrioventricular nodal reentry, which abruptly deteriorated to a multiform ventricular tachycardia. She had not received any antiarrhythmic drugs nor did she have any of the disorders that are usually associated with this atypical ventricular tachycardia.

To our knowledge, the association between multiform ventricular tachycardia and atrioventricular reentrant tachycardia has not been described before.

\section{Case report}

The patient is an 18 year old woman with an eight year history of weekly episodes of rapid palpitation, lasting between half and six hours. On one occasion these had caused syncope. Her father had died suddenly and was known to have had hypertrophic cardiomyopathy. There was no other relevant family history.

For the first time, an electrocardiogram was

Requests for reprints to Dr David H Bennett, Regional Cardiac Centre, Wythenshawe Hospital, Manchester M23 9LT. recorded during palpitation when she recently presented to a casualty department. It showed a regular tachycardia with narrow ventricular complexes at a rate of 188 beats/min. During the recording the rhythm abruptly changed to a multiform ventricular tachycardia (fig 1), which lasted for 30 seconds, after which sinus rhythm returned (fig 2). The patient did not experience dizziness or syncope. Neither oral nor parenteral antiarrhythmic drugs had been administered. She was taking an oral contraceptive but no other drugs.

Electrocardiograms recorded during sinus rhythm were normal. The QTc interval $(0.42 \mathrm{~s})$ was towards the upper limit of normal. Echocardiography was normal and there was no suggestion of hypertrophic cardiomyopathy: during diastole the interventricular septum and posterior left ventricular wall were both $1.0 \mathrm{~cm}$ thick.

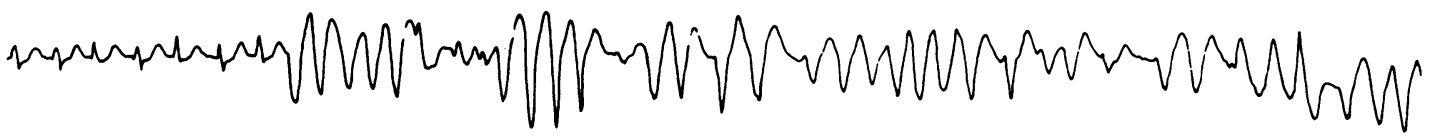

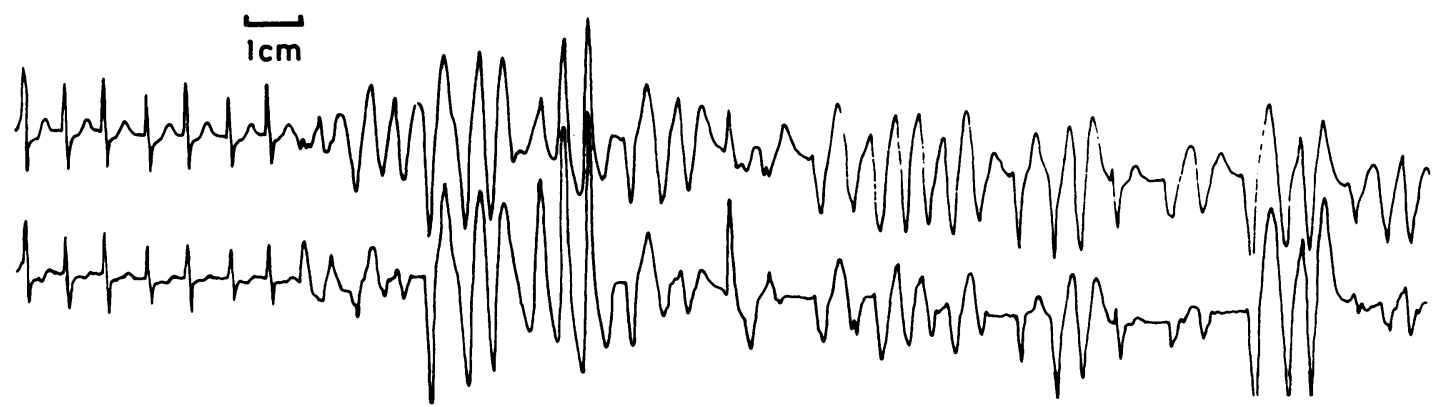

Fig 1 Electrocardiogram with leads I, II, and III recorded simultaneously at $25 \mathrm{~mm} / \mathrm{s}$. Supraventricular tachycardia abruptly changed to multiform ventricular tachycardia. 


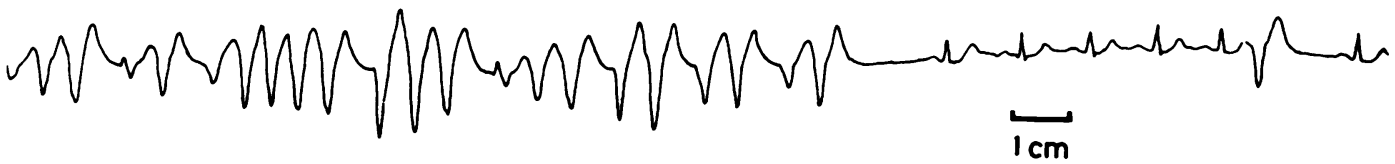

Fig 2 Electrocardiogram showing restoration of sinus rhythm 30 seconds after initiation of multiform ventricular tachycardia.

An intracardiac electrophysiological test was carried out. No evidence of an accessory atrioventricular pathway was found: during ventricular pacing, the ventriculoatrial conduction time increased progressively with increasing prematurity of the ventricular stimulation. Atrioventricular reentrant tachycardia was repeatedly initiated and terminated by precisely timed premature atrial extrastimuli. During tachycardia, the earliest evidence of atrial activation was seen in the His bundle electrogram and coincided with ventricular depolarisation, suggesting that tachycardia was caused by atrioventricular nodal reentry. Multiform ventricular tachycardia did not arise at the termination of the supraventricular tachycardia and was not provoked by rapid atrial pacing or by single and double premature stimuli introduced at the right ventricular apex. The supraventricular tachycardia was repeatedly terminated by a scanning antitachycardia pacemaker that introduced a series of seven premature atrial stimuli: ventricular tachycardia was not precipitated.

She did not tolerate oral sotalol, and oral verapamil in a dose of $\mathbf{4 0} \mathrm{mg}$ three times a day and flecainide $100 \mathrm{mg}$ twice a day were ineffective. The patient refused further antiarrhythmic drugs. A scanning anti-tachycardia atrial pacemaker was implanted (PASAR 4171, Telectronics). Since implantation, episodes of palpitation have been brief and no ventricular arrhythmias have been seen during ambulatory electrocardiography.

\section{Discussion}

Multiform ventricular tachycardia may be caused by abnormalities in ventricular repolarisation, by bradycardias, and by antiarrhythmic drugs ${ }^{1}$ (when it is termed "torsade de pointes"), and also by myocardial infarction. ${ }^{2}$ It has not previously been described in association with atrioventricular reentrant tachycardia. It is noteworthy that ventricular tachycardia was precipitated on only one occasion by the supraventricular tachycardia.

Supraventricular extrasystoles have been reported to initiate uniform ventricular tachycardia ${ }^{3}$ and, after acute myocardial infarction, ventricular fibrillation. ${ }^{4}$ Multiform ventricular tachycardia has been initiated in patients with anterograde ventricular preexcitation ${ }^{5}$ and ventricular fibrillation has been reported in two patients with hypertrophic cardiomyopathy and associated accessory atrioventricular pathways. ${ }^{6}$ But there was no evidence of myocardial infarction, pre-excitation, or hypertrophic cardiomyopathy in our patient in whom the mechanism of initiation of multiform ventricular tachycardia during atrioventricular tachycardia is unclear.

We thank Dr J Hall for his help and for referral of this case.

\section{References}

1 Krikler DM, Curry PVL. Torsade de pointes, an atypical ventricular tachycardia. Br Heart J 1976;38:117-20.

2 Grenadier E, Alpan G, Maor N, et al. Polymorphous ventricular tachycardia in acute myocardial infarction. Am J Cardiol 1984;53:1280-3.

3 Zipes DP, Foster PR, Tromp PJ, Pedersen DH. Atrial induction of ventricular tachycardia: reentry versus triggered automaticity. Am J Cardiol 1979;44:1-8.

4 Bekheit S, Turitto G, Fontaine J, El-Sherif N. Initiation of ventricular fibrillation by supraventricular beats in patients with acute myocardial infarction. $\mathrm{Br}$ Heart J 1988;59:190-5.

5 Brembilla-Perrot B, Terrier de la Chaise A, Isaaz K, Marçon F, Cherrier F, Pernot C. Inducible multiform ventricular tachycardia in Wolff-ParkinsonWhite syndrome. Br Heart J 1987;58:89-95.

6 Krikler DM, Davies MJ, Rowland E, Goodwin JF, Evans RC, Shaw DB. Sudden death in hypertrophic cardiomyopathy: associated accessory atrioventricular pathways. Br Heart J 1980;43:245-51. 